\title{
A NOTE ON WU-ZHENG'S SPLITTING CONJECTURE
}

\author{
CHENGJIE YU
}

(Communicated by Lei $\mathrm{Ni}$ )

\begin{abstract}
Cao's splitting theorem says that for any complete Kähler-Ricci flow $(M, g(t))$ with $t \in[0, T), M$ simply connected and nonnegative bounded holomorphic bisectional curvature, $(M, g(t))$ is holomorphically isometric to $\mathbb{C}^{k} \times(N, h(t))$, where $(N, h(t))$ is a Kähler-Ricci flow with positive Ricci curvature for $t>0$. In this article, we show that $k=n-r$, where $r$ is the Ricci rank of the initial metric. As a corollary, we also confirm a splitting conjecture of $\mathrm{Wu}$ and Zheng when curvature is assumed to be bounded.
\end{abstract}

\section{INTRODUCTION}

In [1], Cao proved the following splitting theorem.

Theorem 1.1 ([1]). Let $g(t)$ with $t \in[0, T)$ be a complete solution to the KählerRicci flow

$$
\frac{d}{d t} g_{i \bar{j}}=-R_{i \bar{j}}
$$

on a complex manifold $M^{n}$ and the initial metric $g(0)$ has nonnegative and bounded holomorphic bisectional curvature. Then, rank $R_{i \bar{j}}(x, t)$ is independent of $x \in M$ and $t \in(0, T)$. Moreover, $\operatorname{ker} R_{i \bar{j}}(x, t)$ is independent of $t>0$ and is a parallel distribution for all $t>0$. In particular, if $M$ is simply connected, $(M, g(t))$ is holomorphically isometric to $\mathbb{C}^{n-k} \times\left(N^{k}, h(t)\right)$, where $h(t)$ with $t \in[0, T)$ is a complete solution to the Kähler-Ricci flow (1.1) on $N$ with positive Ricci curvature for $t>0$.

Let $(M, g)$ be a complete Kähler manifold and $r=\max _{x \in M} \operatorname{rank} R_{i \bar{j}}(x)$. Then $r$ is called the Ricci rank of the Kähler metric $g$. In this article, we show that the number $k$ in Cao's splitting theorem equals the Ricci rank of the initial metric $g(0)$.

Theorem 1.2. Let the settings be the same as in Theorem 1.1 and $r$ be the Ricci rank of the initial metric $g(0)$. Then

$$
\operatorname{rank} R_{i \bar{j}}(x, t)=r
$$

for any $x \in M$ and $t \in(0, T)$.

Received by the editors September 3, 2011.

2010 Mathematics Subject Classification. Primary 53C44; Secondary 53C55.

Key words and phrases. Kähler manifolds, bisectional curvature, splitting theorem.

The author's research was partially supported by the National Natural Science Foundation of China (11001161) and (10901072). 
In [6], Wu and Zheng presented the following splitting conjecture:

Conjecture $1\left([6)\right.$. Let $M^{n}$ be a complete Kähler manifold with nonnegative holomorphic bisectional curvature. Then, its universal covering manifold $\tilde{M}$ is holomorphically isometric to $\mathbb{C}^{n-r} \times N^{r}$, where $r$ is the Ricci rank.

This is a noncompact generalization of the splitting theorem of Howard-Smyth$\mathrm{Wu}$ [3] on compact Kähler manifolds with nonnegative holomorphic bisectional curvature which is also related to a conjecture proposed by Yau (see [6, 7]). In [6] and [7, Wu-Zheng showed that the conjecture is true for complete Kähler manifolds with nonnegative holomorphic bisectional curvature, real analytic Kähler metric and Ricci rank $\leq 2$. The technique used by Wu-Zheng is delicate. They performed some careful analysis on the distribution defined by the kernel of the Ricci tensor. Because the distribution is not defined all over the manifold, they have to require that the metric is real analytic, so that the distribution can be extended in a parallel manner to the whole manifold. As a direct corollary of Theorem 1.1 and Theorem 1.2. we can see that the conjecture is true if curvature is assumed to be bounded.

Theorem 1.3. Let $M^{n}$ be a complete Kähler manifold with nonnegative and bounded holomorphic bisectional curvature. Then, its universal covering manifold $\tilde{M}$ is holomorphically isometric to $\mathbb{C}^{n-r} \times N^{r}$, where $r$ is the Ricci rank.

In 4], Ni and Tam obtained splitting results of complete Kähler manifolds with nonnegative holomorphic bisectional curvature in a similar spirit to Cao's splitting theorem. However, Ni-Tam's splitting results are with respect to function theory on the Kähler manifold. For example, they obtained the following result.

Theorem 1.4 (4]). Let $M$ be a complete simply connected noncompact Kähler manifold with nonnegative holomorphic bisectional curvature and supporting a smooth strictly plurisubharmonic function $u$ on $M$ with bounded gradient. Then $M=\mathbb{C}^{l} \times M_{1} \times M_{2}$ isometrically and holomorphically for some $l \geq 0$, where $M_{1}$ and $M_{2}$ are complete noncompact Kähler manifolds with nonnegative holomorphic bisectional curvature such that any polynomial growth holomorphic function on $M$ is independent of the factor $M_{2}$, and any linear growth holomorphic function is independent of the factors $M_{1}$ and $M_{2}$.

As a direct corollary of Theorem 1.3. we know that $l$ equals the Ricci rank of the manifold if we further assume that the curvature of the manifold is bounded.

Corollary 1.1. Let the settings be the same as in Theorem 1.4 and further assume that the curvature of $M$ is bounded. Then $l$ equals the Ricci rank of the manifold in the conclusion of Theorem 1.4.

\section{Proof of Theorem 1.2}

In this section, using a result of Ferus 2 on the completeness of the leaves of the nullity foliation of the curvature operator, we give a proof of Theorem 1.2 .

Proof of Theorem 1.2. Let $\Omega=\left\{x \in M \mid r=\operatorname{rank} R_{i \bar{j}}(x, 0)\right\}$ and $L_{x}=\operatorname{ker} R_{i \bar{j}}(x, 0)$ for each $x \in \Omega$. It is shown in [6] that $L$ is a totally geodesic and flat foliation on $\Omega$. In 2, Ferus showed that each leaf of the foliation $L$ is complete. Let $F_{x}=\operatorname{ker} R_{i \bar{j}}(x, t)$ for each $x \in M$ and $t>0$. It is shown by Cao's splitting theorem 
(Theorem 1.1) that $F$ is independent of $t$ and parallel for each $t>0$. By continuity (see [5]), we know that $F \subset L$ on $\Omega$ and $F$ is also parallel with respect to $g(0)$. It suffices to show that $L \subset F$ on $\Omega$.

Since $L$ is totally geodesic, flat and with complete leaves, we know that a leaf of $L$ is a totally geodesic and isometric holomorphic immersion $\phi: \mathbb{C}^{n-r} \rightarrow M$. Note that on a Kähler-Ricci flow,

$$
\frac{d}{d t} R i c=\sqrt{-1} \partial \bar{\partial} R
$$

where Ric $=\sqrt{-1} R_{i \bar{j}} d z \wedge d z^{\bar{j}}$ is the Ricci form. Therefore, for each $t>0$,

$$
\operatorname{Ric}(t)=\operatorname{Ric}(0)+\sqrt{-1} \partial \bar{\partial} \int_{0}^{t} R(s) d s
$$

and

$$
\phi^{*} \operatorname{Ric}(t)=\phi^{*} \operatorname{Ric}(0)+\sqrt{-1} \partial \bar{\partial} \int_{0}^{t} R(s)(\phi) d s .
$$

Note that $\phi^{*} \operatorname{Ric}(0)=0$ since $\phi: \mathbb{C}^{n-r} \rightarrow M$ is a leaf of $L$. Hence

$$
\phi^{*} \operatorname{Ric}(t)=\sqrt{-1} \partial \bar{\partial} \int_{0}^{t} R(s)(\phi) d s .
$$

Note that $\operatorname{Ric}(t)$ is nonnegative, so $\int_{0}^{t} R(s)(\phi) d s$ is a bounded pluri-subharmonic function on $\mathbb{C}^{n-r}$. By the Liouville property of pluri-subharmonic functions on complex Euclidean spaces (see [4, Theorem 0.2]), we know that $\int_{0}^{t} R(s)(\phi) d s$ is a constant function. Substituting this back into (2.4), we know that $\phi^{*} \operatorname{Ric}(t)=0$ for $t>0$. Therefore $L \subset F$ on $\Omega$.

Remark 2.1. Instead of using parallel translation to extend the Ricci kernel, here we use the Kähler-Ricci flow to extend the Ricci kernel.

\section{REFERENCES}

[1] Cao, H.-D. On dimension reduction in the Kähler-Ricci flow. Comm. Anal. Geom. 12 (2004), no. 1-2, 305-320. MR2074880(2005d:53104)

[2] Ferus, D. On the completeness of nullity foliations. Michigan Math. J. 18 (1971) 61-64. MR0279733 (43:5454)

[3] Howard, A.; Smyth, B.; Wu, H. On compact Kähler manifolds of nonnegative bisectional curvature. I. Acta Math. 147 (1981), no. 1-2, 51-56. MR631087 (83e:53064a)

[4] Ni, L.; Tam, L.-F. Plurisubharmonic functions and the structure of complete Kähler manifolds with nonnegative curvature. J. Differential Geom. 64 (2003), no. 3, 457-524. MR2032112 (2005a:32023)

[5] Shi, Wan-Xiong. Deforming the metric on complete Riemannian manifolds. J. Differential Geom. 30 (1989), no. 1, 223-301. MR1001277 (90i:58202)

[6] Wu, H.; Zheng, F. Kähler manifolds with slightly positive bisectional curvature. Explorations in complex and Riemannian geometry, 305-325, Contemp. Math., 332, Amer. Math. Soc., Providence, RI, 2003. MR2018347 (2005d:32043)

[7] Wu, H.; Zheng, F. Compact Kähler manifolds with nonpositive bisectional curvature. J. Differential Geom. 61 (2002), no. 2, 263-287. MR.1972147(2004b:53128)

Department of Mathematics, Shantou University, Shantou, Guangdong, People's Republic of China

E-mail address: cjyu@stu.edu.cn 